\title{
Vitamin $D$ and Vitamin D binding protein: the inseparable duo in COVID-19
}

\author{
M. M. Speeckaert ${ }^{1,2} \odot \cdot$ R. Speeckaert ${ }^{2,3} \cdot$ J. R. Delanghe ${ }^{4}$
}

Received: 14 March 2021 / Accepted: 6 April 2021 / Published online: 11 April 2021

(C) Italian Society of Endocrinology (SIE) 2021

Keywords COVID-19 · Polymorphism · Vitamin D binding protein · Vitamin D deficiency

To the editor,

With interest, we read the paper of Mazziotti et al. [1], which showed that vitamin D deficiency, when associated with secondary hyperparathyroidism, may negatively impact the clinical outcome of severe acute respiratory syndrome coronavirus 2 (SARS-CoV-2)-related pneumonia. As vitamin D binding protein (DBP) binds the majority (85-90\%) of vitamin D metabolites, we would like to highlight the potential influence of this major plasma carrier protein and its polymorphism on the reported results.

DBP is a highly polymorphic serum protein, determined by two common SNPs (rs7041 and rs4588) and three DBP isotypes (DBP1F, DBP1S, and DBP2). We have demonstrated an association between the DBP 1 allele frequency and a lower prevalence and mortality due to a SARS-CoV-2 infection [2]. The plasma concentrations of vitamin D metabolites and DBP are highest in DBP1-1 subjects, intermediate in DBP1-2, and lowest in DBP2-2. In healthy adults, the relationship between PTH and 25(OH)-vitamin D is little affected by DBP until the extremes of serum DBP concentrations and vitamin D intake are reached. Although there is no direct evidence of a link between vitamin D concentrations and coronavirus disease-19 (COVID-19) incidence or outcomes, the immunomodulatory role of vitamin $\mathrm{D}$ in

M. M. Speeckaert

Marijn.Speeckaert@ugent.be

1 Department of Nephrology, Ghent University Hospital, Corneel Heymanslaan 10 9000, Ghent, Belgium

2 Research Foundation-Flanders (FWO), Brussels, Belgium

3 Department of Dermatology, Ghent University Hospital, Ghent, Belgium

4 Department of Diagnostic Sciences, Ghent University, Ghent, Belgium respiratory infections could deliver some indirect arguments why DBP1-carriers have a better prognosis. However as only $1-2 \%$ of its sterol binding sites are utilized, multiple other metabolic roles of DBP could play a role in the course of COVID-19 such as actin scavenging, and modulation of inflammatory processes and innate immunity.

Critical hypoxemia fulfilling the criteria of acute respiratory distress syndrome (ARDS) is observed in approximately $20 \%$ of symptomatic patients with COVID-19. ARDS, multiple organ dysfunction syndrome, and septic shock are characterized by actin release which is involved in microvascular impairment. A catastrophic, complement-mediated thrombotic microvascular injury occurs in severe COVID-19 infection, with sustained activation of the actin pathway and lectin pathway cascades. In comparison to healthy volunteers, the administration of plasma from COVID-19 patients resulted in a disruption of the human pulmonary microvascular endothelial cell monolayer with loss of junctional VE-cadherin and cortical actin, the formation of actin stress fibers, and inter-endothelial gap formation [3]. The rapid transformation of actin monomers into polymeric structures could result in clogging in the microcirculation, which can be countered by the actin scavenger system, consisting of DBP and gelsolin. DBP binds with very high affinity to actin in a one-to-one complex and the DBP-actin complexes are more rapidly cleared from the circulation than DBP alone. The value of low serum DBP concentration as a marker for disease severity has previously been demonstrasted and has prognostic significance in patients with severe illness.

Besides its role as an actin scavenger, DBP is the precursor of the immunomodulatory protein, DBP-macrophage activating factor (DBP-MAF). In the first phase, DBP-MAF can activate macrophages at sites of infection/inflammation and in a second phase, it can induce their apoptosis by upregulating caspase activity via the $\mathrm{p} 38$ and JNK1/2 pathways. DBP-MAF promotes also the production of nitric 
oxide, which has therapeutic potential to mitigate COVID19 severity.

In conclusion, the multifunctional character of DBP and its polymorphism could play an important role in the pathogenesis of COVID-19.

\section{Declarations}

Conflict of interest All the authors do not have confict of interest that is relevant to the subject matter or materials included in this work.

\section{References}

1. Mazziotti G, Lavezzi E, Brunetti A, Mirani M, Favacchio G, Pizzocaro A, Sandri MT, Di Pasquale A, Voza A, Ciccarelli M,
Lania AG, Humanitas COVID19 Task Force (2021) Vitamin D deficiency, secondary hyperparathyroidism and respiratory insufficiency in hospitalized patients with COVID-19. J Endocrinol Invest (in press). https://doi.org/10.1007/s40618-021-01535-2

2. Speeckaert MM, De Buyzere ML, Delanghe JR (2021) Vitamin D binding protein polymorphism and COVID-19. J Med Virol 93(2):705-707. https://doi.org/10.1002/jmv.26508

3. Michalick L, Weidenfeld S, Grimmer B, Fatykhova D, Solymosi PD, Behrens F, Dohmen M, Brack MC, Schulz S, Thomasch E, Simmons S, Müller-Redetzky H, Suttorp N, Kurth F, Neudecker J, Toennies M, Bauer TT, Eggeling S, Corman VM, Hocke AC, Witzenrath M, Hippenstiel S, Kuebler WM (2021) Plasma mediators in patients with severe COVID-19 cause lung endothelial barrier failure. EurRespir J 57(3):2002384. https://doi.org/10.1183/13993 003.02384-2020

Publisher's Note Springer Nature remains neutral with regard to jurisdictional claims in published maps and institutional affiliations. 